\title{
Geometric properties of mixed operator involving Ruscheweyh derivative and Sălăgean operator
}

\author{
Rabha W. Ibrahim, Mayada T. Wazi and Nadia Al-Saidi
}

\begin{abstract}
Operator theory is a magnificent tool for studying the geometric behaviors of holomorphic functions in the open unit disk. Recently, a combination between two well known differential operators, Ruscheweyh derivative and Sălăgean operator are suggested by Lupas in [10]. In this effort, we shall follow the same principle, to formulate a generalized differential-difference operator. We deliver a new class of analytic functions containing the generalized operator. Applications are illustrated in the sequel concerning some differential subordinations of the operator.
\end{abstract}

Mathematics Subject Classification (2010): 30C45.

Keywords: Differential operator, conformable operator, fractional calculus, unit disk, univalent function, analytic function, subordination and superordination.

\section{Introduction}

Differential operators in a complex domain play a significant role in functions theory and its information. They have used to describe the geometric interpolation of analytic functions in a complex domain. Also, they have utilized to generate new formulas of holomorphic functions. Lately, Lupas [10] presented a amalgamation of two well-known differential operators prearranged by Ruscheweyh [12] and Sălăgean [13]. Later, these operators are investigated by researchers considering different classes and formulas of analytic functions $[5,8]$.

In this note, we consider a special class of functions in the open unit disk

$$
\sqcup=\{\xi \in \mathbb{C}|| \xi \mid<1\}
$$

denoting by $\Sigma$ and having the series

$$
\varphi(\xi)=\xi+\sum_{n=2}^{\infty} \varphi_{n} \xi^{n}, \quad \xi \in \sqcup .
$$


Let $\varphi \in \Sigma$, then the Ruscheweyh formula is indicated by the structure formula

$$
\Phi^{m} \varphi(\xi)=\xi+\sum_{n=2}^{\infty} C_{m+n-1}^{m} \varphi_{n} \xi^{n} .
$$

While, the Sălăgean operator admits the construction

$$
\Psi^{m} \varphi(\xi)=\xi+\sum_{n=2}^{\infty} n^{m} \varphi_{n} \xi^{n}
$$

Lupas operator is formulated by the structure

$$
\lambda_{\sigma}^{m} \varphi(\xi)=\xi+\sum_{n=2}^{\infty}\left[\sigma n^{m}+(1-\sigma) C_{m+n-1}^{m}\right] \varphi_{n} \xi^{n}, \quad \xi \in \sqcup, \sigma \in[0,1] .
$$

Newly, Ibrahim and Darus [7] considered the next differential operator

$$
\begin{aligned}
\Theta_{\kappa}^{0} \varphi(\xi) & =\varphi(\xi) \\
\Theta_{\kappa}^{1} \varphi(\xi) & =\xi \varphi(\xi)^{\prime}+\frac{\kappa}{2}(\varphi(\xi)-\varphi(-\xi)-2 \xi), \quad \kappa \in \mathbb{R} \\
\vdots & \\
\Theta_{\kappa}^{m} \varphi(\xi) & =\Theta_{\kappa}\left(\Theta_{\kappa}^{m-1} \varphi(\xi)\right) \\
& =\xi+\sum_{n=2}^{\infty}\left[n+\frac{\kappa}{2}\left(1+(-1)^{n+1}\right)\right]^{m} \varphi_{n} \xi^{n} .
\end{aligned}
$$

When $\kappa=0$,we have $\Psi^{m} \varphi(\xi)$ In addition, it is a modified formula of the well-known Dunkl operator [2], where $\kappa$ is known as the Dunkl order. Proceeding, we define a generalized formula of $\lambda_{\sigma}^{m}$, as follows:

$$
\begin{aligned}
J_{\sigma, \kappa}^{m} \varphi(\xi) & =(1-\sigma) \Phi^{m} \varphi(\xi)+\sigma \Theta_{\kappa}^{m} \varphi(\xi) \\
& =\xi+\sum_{n=2}^{\infty}\left[(1-\sigma) C_{m+n-1}^{m}+\sigma\left(n+\frac{\kappa}{2}\left(1+(-1)^{n+1}\right)\right)^{m}\right] \varphi_{n} \xi^{n} .
\end{aligned}
$$

Clearly, the operator $J_{\sigma, \kappa}^{m} \varphi(\xi) \in \Sigma$.

\section{Remark 1.1.}

- $m=0 \Longrightarrow J_{\sigma, \kappa}^{0} \varphi(\xi)=\varphi(\xi)$;

- $\kappa=0 \Longrightarrow J_{\sigma, 0}^{m} \varphi(\xi)=\lambda_{\sigma}^{m} \varphi(\xi)$;

- $\sigma=0 \Longrightarrow J_{0, \kappa}^{m} \varphi(\xi)=\Phi^{m} \varphi(\xi)$;

- $\sigma=1 \Longrightarrow J_{1, \kappa}^{m} \varphi(\xi)=\Theta_{\kappa}^{m} \varphi(\xi)$;

- $\kappa=0, \sigma=1 \Longrightarrow J_{1,0}^{m} \varphi(\xi)=\Psi^{m} \varphi(\xi)$.

Definition 1.2. Consider the following data $\epsilon \in[0,1), \sigma \in[0,1], \kappa \geq 0$, and $m \in \mathbb{N}$. Then a function $\varphi \in \Sigma$ belongs to the set $\top_{m}(\sigma, \kappa, \epsilon)$ if and only if

$$
\Re\left(\left(J_{\sigma, \kappa}^{m} \varphi(\xi)\right)^{\prime}\right)>\epsilon, \quad \xi \in \sqcup .
$$


Observe that the set $\top_{m}(\sigma, \kappa, \epsilon)$ is an extension of the well known class of bounded turning functions (see [1]-[14]). Next results are requested to prove our results depending on the subordination concept (see [11]).

Lemma 1.3. Suppose that $\hbar$ is convex function such that $\hbar(0)=b$, and there is a complex number with a positive real part $\mu$. If $b \in \mathfrak{H}[b, n]$, where

$$
\mathfrak{H}[b, n]=\left\{b \in \mathfrak{H}: b(\xi)=b+b_{n} \xi^{n}+b_{n+1} \xi^{n+1}+\ldots\right\}
$$

(the space of holomorphic functions) and

$$
b(\xi)+\frac{1}{\mu} \xi b^{\prime}(\xi) \prec \hbar(\xi), \quad \xi \in \sqcup,
$$

then

$$
b(\xi) \prec \iota(\xi) \prec \hbar(\xi),
$$

with

$$
\iota(\xi)=\frac{\mu}{n \xi^{\mu / n}} \int_{0}^{\xi} \hbar(\tau) \tau^{\frac{\mu}{(n-1)}} d \tau, \quad \xi \in \sqcup .
$$

Lemma 1.4. Suppose that the convex function $b(\xi)$ satisfies the functional

$$
\hbar(\xi)=b(\xi)+n \mu\left(\xi b^{\prime}(\xi)\right)
$$

for $\mu>0$ and $n$ is a positive integer. If $b \in \mathfrak{H}[\hbar(0), n]$, and $b(\xi)+\mu \xi b^{\prime}(\xi) \prec \hbar(\xi), \xi \in \sqcup$ then $b(\xi) \prec \hbar(\xi)$, and this outcome is sharp.

Lemma 1.5. (i) If $\lambda>0, \gamma>0, \beta=\beta(\gamma, \lambda, n)$ and $b \in \mathfrak{H}[1, n]$ then

$$
b(\xi)+\lambda \xi b^{\prime}(\xi) \prec\left[\frac{1+\xi}{1-\xi}\right]^{\beta} \Rightarrow b(\xi) \prec\left[\frac{1+\xi}{1-\xi}\right]^{\gamma} .
$$

(ii) If $\epsilon \in[0,1), \lambda=\lambda(\epsilon, n)$ and $b \in \mathfrak{H}[1, n]$ then

$$
\Re\left(b^{2}(\xi)+2 b(\xi) \cdot \xi b^{\prime}(\xi)\right)>\epsilon \Rightarrow \Re(b(\xi))>\lambda .
$$

\section{Results}

In this section, we investigate some geometric conducts of the operator (1.1).

Theorem 2.1. The set $\top_{m}(\sigma, \kappa, \epsilon)$ is convex.

Proof. Suppose that $\varphi_{i}, i=1,2$ are two functions belonging to $\top_{m}(\sigma, \kappa, \epsilon)$ satisfying

$$
\varphi_{1}(\xi)=\xi+\sum_{n=2}^{\infty} \varphi_{n} \xi^{n}
$$

and

$$
\varphi_{2}(\xi)=\xi+\sum_{n=2}^{\infty} \phi_{n} \xi^{n} .
$$

It is sufficient to prove that the function

$$
\Pi(x 1)=\wp_{1} \varphi_{1}(\xi)+\wp_{2} \varphi_{2}(\xi), \quad \xi \in \sqcup
$$


is in $\top_{m}(\sigma, \kappa, \epsilon)$, where $\wp_{1}>0, \wp_{2}>0$ and $\wp_{1}+\wp_{2}=1$. The formula of $\Pi(z)$ yields

$$
\Pi(\xi)=\xi+\sum_{n=2}^{\infty}\left(\wp_{1} \varphi_{n}+\wp_{2} \phi_{n}\right) \xi^{n} .
$$

Thus, under the operator (1.1), we get

$$
J_{\sigma, \kappa}^{m} \Pi(\xi)=\xi+\sum_{n=2}^{\infty}\left(\wp_{1} \varphi_{n}+\wp_{2} \phi_{n}\right)\left[(1-\sigma) C_{m+n-1}^{m}+\sigma\left(n+\frac{\kappa}{2}\left(1+(-1)^{n+1}\right)\right)^{m}\right] \xi^{n} .
$$

By making a differentiation, we obtain

$$
\begin{gathered}
\Re\left\{\left(J_{\alpha, \kappa}^{m} \Pi(\xi)\right)^{\prime}\right\} \\
=1+\wp_{1} \Re\left\{\sum_{n=2}^{\infty} n\left[(1-\sigma) C_{m+n-1}^{m}+\sigma\left(n+\frac{\kappa}{2}\left(1+(-1)^{n+1}\right)\right)^{m}\right] \varphi_{n} \xi^{n-1}\right\} \\
+\wp_{2} \Re\left\{\sum_{n=2}^{\infty} n\left[(1-\sigma) C_{m+n-1}^{m}+\sigma\left(n+\frac{\kappa}{2}\left(1+(-1)^{n+1}\right)\right)^{m}\right] \phi_{n} \xi^{n-1}\right\}=\epsilon .
\end{gathered}
$$

Theorem 2.2. Define the following functions: $\varphi \in \top_{m}(\sigma, \kappa, \epsilon), \phi$ be convex and

$$
F(\xi)=\frac{2+c}{\xi^{1+c}} \int_{0}^{\xi} t^{c} \varphi(t) d t, \quad \xi \in \sqcup .
$$

Then

$$
\left(J_{\alpha, \kappa}^{m} \varphi(\xi)\right)^{\prime} \prec \phi(\xi)+\frac{\left(\xi \phi^{\prime}(\xi)\right)}{2+c}, \quad c>0
$$

yields

and this outcome is sharp.

$$
\left(J_{\sigma, \kappa}^{m} F(\xi)\right)^{\prime} \prec \phi(\xi)
$$

Proof. By the assumptions, we have

$$
\left(J_{\sigma, \kappa}^{m} F(\xi)\right)^{\prime}+\frac{\left(J_{\sigma, \kappa}^{m} F(\xi)\right)^{\prime \prime}}{2+c}=\left(J_{\sigma, \kappa}^{m} \varphi(\xi)\right)^{\prime} .
$$

Consequently, we get

$$
\left(J_{\sigma, \kappa}^{m} F(\xi)\right)^{\prime}+\frac{\left(J_{\sigma, \kappa}^{m} F(\xi)\right)^{\prime \prime}}{2+c} \prec \phi(\xi)+\frac{\left(\xi \phi^{\prime}(\xi)\right)}{2+c} .
$$

Assuming

one can find

$$
b(\xi):=\left(J_{\sigma, \kappa}^{m} F(\xi)\right)^{\prime},
$$

$$
b(\xi)+\frac{\left(\xi b^{\prime}(\xi)\right)}{2+c} \prec \phi(\xi)+\frac{\left(\xi \phi^{\prime}(\xi)\right)}{2+c} .
$$

In virtue of Lemma 1.3, we have

$$
\left(J_{\sigma, \kappa}^{m} F(\xi)\right)^{\prime} \prec \phi(\xi),
$$


and $\phi$ is the best dominant.

Theorem 2.3. Assume the convex function $\phi$ achieving $\phi(0)=1$ and for $\varphi \in \Sigma$

$$
\left(J_{\sigma, \kappa}^{m} \varphi(\xi)\right)^{\prime} \prec \phi(\xi)+\xi \phi^{\prime}(\xi), \quad \xi \in \sqcup,
$$

then

$$
\frac{J_{\sigma, \kappa}^{m} \varphi(\xi)}{\xi} \prec \phi(\xi),
$$

and this outcome is sharp.

Proof. Formulate the next functional

$$
b(z):=\frac{J_{\sigma, \kappa}^{m} \varphi(\xi)}{\xi} \in \mathfrak{H}[1,1]
$$

Consequently, we get

$$
J_{\sigma, \kappa}^{m} \varphi(\xi)=\xi b(\xi) \Longrightarrow\left(J_{\sigma, \kappa}^{m} \varphi(\xi)\right)^{\prime}=b(\xi)+\xi b^{\prime}(\xi) .
$$

Therefore, we obtain the inequality

$$
b(\xi)+\xi b^{\prime}(\xi) \prec \phi(\xi)+\xi \phi^{\prime}(\xi) .
$$

According to Lemma 1.4, we attain

$$
\frac{J_{\sigma, \kappa}^{m} \varphi(\xi)}{\xi} \prec \phi(\xi),
$$

and $\phi$ is the best dominant.

Theorem 2.4. For $\varphi \in \Sigma$ if the inequality

$$
\left(J_{\sigma, \kappa}^{m} \varphi(\xi)\right)^{\prime} \prec\left(\frac{1+\xi}{1-\xi}\right)^{\beta}, \quad \xi \in \sqcup, \beta>0,
$$

achieves then

$$
\Re\left(\frac{J_{\sigma, \kappa}^{m} \varphi(\xi)}{\xi}\right)>\epsilon
$$

for some $\epsilon \in[0,1)$.

Proof. For the function $b(\xi)$ in (2.1), we have

$$
\left(J_{\sigma, \kappa}^{m} \varphi(\xi)\right)^{\prime}=\xi b^{\prime}(\xi)+b(\xi) \prec\left(\frac{1+\xi}{1-\xi}\right)^{\beta} .
$$

According to Lemma 1.5.i, there occurs a constant $\gamma>0$ with $\beta=\beta(\gamma)$ with

$$
\frac{J_{\sigma, \kappa}^{m} \varphi(\xi)}{\xi} \prec\left(\frac{1+\xi}{1-\xi}\right)^{\gamma} .
$$

This yields $\Re\left(J_{\sigma, \kappa}^{m} \varphi(\xi) / \xi\right)>\epsilon$, for some $\epsilon \in[0,1)$. 
Theorem 2.5. Assume that $\varphi \in \Sigma$ achieves the inequality

$$
\Re\left(\left(J_{\sigma, \kappa}^{m} \varphi(\xi)\right)^{\prime} \frac{J_{\sigma, \kappa}^{m} \varphi(\xi)}{\xi}\right)>\frac{\sigma}{2}, \quad \xi \in \sqcup, \sigma \in[0,1) .
$$

Then $J_{\sigma, \kappa}^{m} \varphi(\xi) \in \top_{m}(\sigma, \kappa, \epsilon)$ for some $\epsilon \in[0,1)$. In addition, it is univalent of bounded turning in $\sqcup$.

Proof. Assume the function $b(\xi)$ as in (2.1). A Calculation implies that

$$
\Re\left(b^{2}(\xi)+2 b(\xi) \cdot \xi b^{\prime}(\xi)\right)=2 \Re\left(\left(J_{\sigma, \kappa}^{m} \varphi(\xi)\right)^{\prime} \frac{J_{\sigma, \kappa}^{m} \varphi(\xi)}{\xi}\right)>\sigma .
$$

Lemma 1.5.ii, implies that there occurs a constant $\lambda(\sigma)$ satisfying $\Re(b(\xi))>\lambda(\sigma)$. Thus, we obtain $\Re(b(\xi))>\epsilon$ for some $\epsilon \in[0,1)$. It yields from (2.2) that $\left.\Re\left(J_{\sigma, \kappa}^{m} \varphi(\xi)\right)^{\prime}\right)>\epsilon$ and by Noshiro-Warschawski and Kaplan Theorems (see [3]), we have that $J_{\sigma, \kappa}^{m} \varphi(\xi)$ is univalent and of bounded turning in $\sqcup$.

\section{References}

[1] Darus, M., Ibrahim, R.W., Partial sums of analytic functions of bounded turning with applications, J. Comput. Appl. Math., 29(2010), 81-88.

[2] Dunkl, C.F., Differential-difference operators associated with reflections groups, Trans. Am. Math. Soc., 311(1989), 167-183.

[3] Duren, P., Univalent Functions, Grundlehren der Mathematischen Wissenschaften, Springer-Verlag New York Inc., 1983.

[4] Ibrahim, R.W., Geometric properties of the differential shift plus complex Volterra operator, Asian-Eur. J. Math., 11.01(2018), 1850013.

[5] Ibrahim, R.W., Generalized Briot-Bouquet differential equation based on new differential operator with complex connections, Gen. Math., 28(2020), 105-114.

[6] Ibrahim, R.W., Darus M., Extremal bounds for functions of bounded turning, Int. Math. Forum, 6(2011), 1623-1630.

[7] Ibrahim, R.W., Darus M., Subordination inequalities of a new Sălăgean-difference operator, Int. J. Math. Comput. Sci., 14(2019), 573-582.

[8] Ibrahim, R.W., Elobaid, R.M., Obaiys, S.J., Generalized Briot-Bouquet differential equation based on new differential operator with complex connections, Axioms, 9(2020), 1-13.

[9] Krishna, D., et al., Third Hankel determinant for bounded turning functions of order alpha, J. Nigerian Math. Soc., 34.2(2015), 121-127.

[10] Lupaş Alb, A., On special differential subordinations using Sălăgean and Ruscheweyh operators, Math. Inequal. Appl., 12(2009), 781-790.

[11] Miller, S.S., Mocanu, P.T., Differential Subordinations: Theory and Applications, CRC Press, 2000.

[12] Ruscheweyh, St., New criteria for univalent functions, Proc. Amer. Math. Soc., 49(1975), 109-115.

[13] Sălăgean, G. St., Subclasses of univalent functions, Lecture Notes in Math., Springer Verlag, Berlin, 1013(1983), 362-372.

[14] Tuneski, N., Bulboacă, T., Sufficient conditions for bounded turning of analytic functions, Ukr. Math. J., 70.8(2019), 1288-1299. 
Rabha W. Ibrahim

IEEE: 94086547, Kuala Lumpur, 59200,

Malaysia

e-mail: rabhaibrahim@yahoo.com

Mayada T. Wazi

University of Technology,

Department of Electromechanical Engineering,

Iraq

e-mail: mayada.t.wazi@uotechnology.edu.iq

Nadia Al-Saidi

University of Technology,

Department of Applied Sciences,

Iraq

e-mail: nadiamg08@gmail.com 